\title{
The Essential Tremor Rating Assessment Scale
}

\author{
Rodger J. Elble
}

Department of Neurology, Southern Illinois University School of Medicine, Springfield, USA

\section{Article Info}

\section{Article Notes}

Received: June 27, 2016

Accepted: July 27, 2016

*Correspondence:

Dr. Rodger J. Elble

Department of Neurology, Southern Illinois University School of

Medicine, PO Box 19645

Springfield, IL 62794-9645, USA

Telephone: $217-545-7194$

E-mail: relble@siumed.edu

() 2016 Elble RJ. This article is distributed under the terms of the Creative Commons Attribution 4.0 International License

\section{Keywords}

Essential Tremor

Rating Scale

Action Tremor

Activities of daily living

\section{ABSTRACT}

The Essential Tremor Rating Assessment Scale was developed by the Tremor Research Group (www.tremorresearchgroup.org) to quantify essential tremor severity and its impact on activities of daily living. This scale requires only a pen and paper, and can be completed in about 10 minutes. Upper extremity action tremor is the main focus of this scale, but action tremor is also assessed in the head, face, voice, and lower limbs. The scale has excellent face validity, inter- and intra-rater reliability, and sensitivity to change. The activities of daily living section correlates strongly with the performance section, and this scale also correlates strongly with transducer measures of tremor and with the Fahn-Tolosa-Marín tremor rating scale. In the Fahn-Tolosa-Marín tremor rating scale, upper extremity tremor greater than $4 \mathrm{~cm}$ corresponds to a maximum rating of 4 , while grade 4 tremor in the Essential Tremor Rating Assessment Scale corresponds to an amplitude greater than $20 \mathrm{~cm}$. Therefore, the Essential Tremor Rating Assessment Scale is better suited for assessment of severe essential tremor.

\section{Introduction}

The Tremor Research Group (TRG) in the United States (www. tremorresearchgroup.org) began developing the Essential Tremor Rating Assessment Scale (TETRAS) in 2003. Until then, the most widely used scale was the Fahn-Tolosa-Marín scale (FTM) ${ }^{1}$, which had undergone limited validation in essential tremor $(E T)^{2}$ and which has upper extremity tremor amplitude anchors that are too low for severe ET. TETRAS was therefore developed in a series of several modifications and validation exercises, and it was ultimately published in $2012^{3}$. This review summarizes the strengths and limitations of TETRAS and provides guidelines for its use by individuals and by commercial entities.

\section{Overview of TETRAS}

TETRAS was designed to provide an accurate, comprehensive quantification of ET in approximately 10 minutes, requiring only a pen and paper. TETRAS has an activities of daily living (ADL) section and a performance section ${ }^{3}$. Both sections emphasize tremor in the upper limbs, which is the primary source of disability in ET. A Microsoft Excel spreadsheet of TETRAS with instructions and scoring are included in the on-line appendix. Instructions for videotaping a TETRAS exam are available upon request from TRG (tremorresearchgroup@gmail.com).

The ADL section of TETRAS has 12 items, each rated $0,1,2,3$ or 4 . The maximum total score is 48 . Item 1 addresses speech; item 10 addresses occupational impairment; and item 12 assesses social 
impact. The other 9 items assess activities that are affected primarily by upper limb tremor (see on-line appendix).

The performance section of TETRAS has 9 items rated 0-4 and a maximum total score of 64. Items 1 (head tremor), 2 (face tremor), 3 (voice tremor), 5 (lower limb tremor), 7 (handwriting), and 9 (standing) have maximum scores of 4. Lower limb tremor (item 5) is assessed bilaterally during posture and heel-knee-shin movement, but only the maximum tremor observed during the four assessments is scored, resulting in an obvious underweighting of lower limb tremor in the total performance score. By contrast, item 4 is the sum of 0-4 ratings of right and left upper limb tremor in three tasks: 1) postural tremor with upper limbs held forward and horizontally, 2) postural tremor with upper limbs extended laterally and horizontally, with the elbows flexed and hands positioned close to each other near the chin ("wing beating position"), and 3) kinetic tremor during finger-nose (or chin)-finger movements. The sum of the three tasks and two extremities results in an item 4 score of 0-24. Similarly, the spiral drawing task (item 6) and the dot approximation task (item 8; a test of upper limb tremor) are scored for the right and left upper limbs and summed to produce item scores of $0-8$. This scoring results in an intentional overweighting of upper limb tremor in TETRAS.

For head, face, voice, lower limbs, spiral drawing, handwriting, and standing (items 1, 2, 3, 5, 6, 7 and 9), the scores are defined by $0-4$ whole numbers. However, 0.5 -point increments may be used if a rating cannot be reconciled to the higher or lower whole number (e.g., a score of 1.5 when the rater is uncertain whether the score is 1 or 2 ). Each 0.5 increment in rating is explicitly defined for upper limb postural and kinetic tremor (item 4) and for the dot approximation task (item 8) (Table 1).

\begin{tabular}{|c|c|c|c|}
\hline Rating & $\begin{array}{c}\text { Upper limb } \\
\text { tremor* }\end{array}$ & $\begin{array}{c}\text { Lower limb } \\
\text { tremor** }\end{array}$ & Head tremor** \\
\hline 0 & none & none & none \\
\hline 0.5 & & & \\
\hline 1 & $<0.5 \mathrm{~cm}$ & $<0.5 \mathrm{~cm}$ & $<0.5 \mathrm{~cm}$ \\
\hline 1.5 & $0.5-<1 \mathrm{~cm}$ & & \\
\hline 2 & $1-<3 \mathrm{~cm}$ & $0.5-<1 \mathrm{~cm}$ & $0.5-<2.5 \mathrm{~cm}$ \\
\hline 2.5 & $3-<5 \mathrm{~cm}$ & & \\
\hline 3 & $5-<10 \mathrm{~cm}$ & $1-<5 \mathrm{~cm}$ & $2.5-5 \mathrm{~cm}$ \\
\hline 3.5 & $10-<20 \mathrm{~cm}$ & & \\
\hline 4 & $\geq 20 \mathrm{~cm}$ & $\geq 5 \mathrm{~cm}$ & $>5 \mathrm{~cm}$ \\
\hline
\end{tabular}

*These anchors are used for the assessment of upper limb tremor in two postures, during finger-nose-finger testing, and in the dot approximation task (TETRAS performance items 4 and 8; see on-line appendix for a detailed description of TETRAS).

**Half point increments are used when the rater is uncertain whether the rating fits the higher or lower whole number. A score of 0.5 is given when the rater is uncertain whether tremor is present.

Table 1: Metric anchors for TETRAS performance ratings of limb and head tremor.
All items of the examination, except standing tremor and heel-knee-shin testing, are performed with the patient seated comfortably. Heel-knee-shin testing is performed in the supine position. For each item, scoring is based on the highest peak-to-peak amplitude seen at any point during the exam. Patients are instructed not to suppress or control their tremor, but to simply perform the task in a manner that permits full expression of tremor severity.

TETRAS does not provide a comprehensive characterization of patients with ET. In particular, there is no assessment of anxiety and depression, which are known to impact the quality of life in $\mathrm{ET}^{4,5}$, and there is no assessment of rest tremor, which is not a typical feature of ET but may occur in severely affected people. Anxiety, depression and quality of life can be assessed with scales designed for these purposes, as recently reviewed ${ }^{4-6}$. Rest tremor was not included because its determination is a common source of diagnostic uncertainty ${ }^{7}$. Postural tremor in severe ET is often misconstrued as rest tremor in a patient that is not given adequate opportunity to relax the affected body part. For example, rest tremor is often assessed while a patient sits in a chair with back unsupported and forearms supinated on the patient's lap. Inadequate support of the torso enables postural tremor in the torso, and supination of the forearms requires active contraction of the forearm supinator muscles. In contrast to rest tremor in Parkinson disease, rest tremor in ET does not subside when the muscles are activated for a voluntary movement and usually does not increase during walking ${ }^{7,8}$.

TETRAS was designed for the clinical assessment of ET in clinical trials and for routine clinical assessment of treatment effects and disease progression. It has excellent face validity, and it correlates strongly with transducer measures of upper limb ${ }^{9,10}$ and head tremor ${ }^{11}$. TETRAS also correlates strongly with the FTM (Figure 1). TETRAS

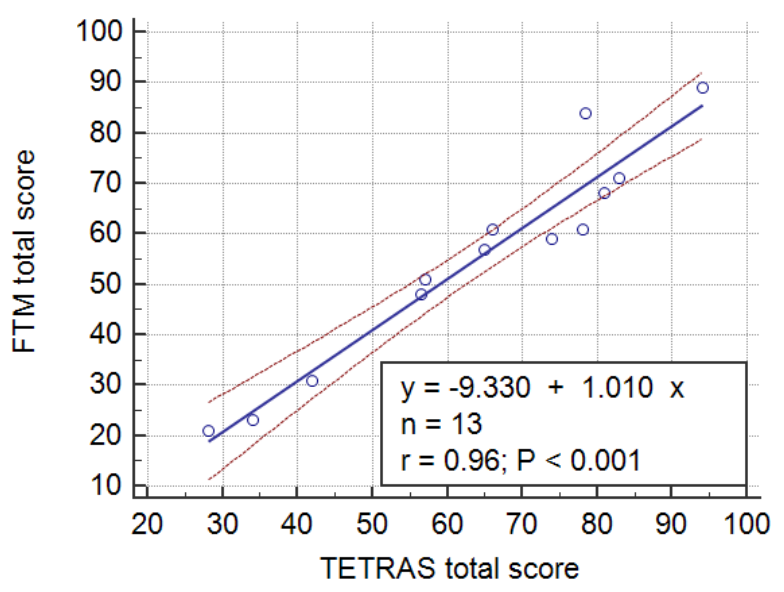

Figure 1: Linear regression and 95\% confidence limits for FTM versus TETRAS total scores obtained from 13 patients ( 7 men) with moderate-severe ET. The two exams were performed less than one hour apart by one of two experienced examiners. 
has a sensitivity to change (minimum detectable change) that is comparable to that of accelerometry, gyroscopy and digitizing tablets ${ }^{11,12}$. This is also true for the FTM ${ }^{11,13-15}$. This surprising result is due to the fact that while transducers are far more precise and accurate than clinical ratings, the advantages of this precision and accuracy are mitigated by the spontaneous random variability in tremor amplitude ${ }^{16}$. Thus, the FTM and TETRAS are comparable to transducers in their ability to detect change that exceeds random variability. TETRAS has been used in clinical trials ${ }^{10,17}$ and has been shown to be sensitive to change in baseline tremor amplitude.

I used TETRAS in the routine clinical assessment of 169 consecutive patients (85 men) referred to my clinic for ET, as defined by the Tremor Investigation Group ${ }^{18}$. Tremor severity in the upper limbs ranged from mild to severe. The TETRAS scores were normally distributed, and summary statistics for these patients are provided in Table 2. Summary statistics for patients with scores above and below the median are also provided. TETRAS ADL and performance scores correlated strongly (Figure 2), as reported previously for a different patient cohort and multiple raters ${ }^{3}$.

Nine of the 169 patients were re-examined by me 2-6 months after the initial exam, with no interval change in therapy. The test-retest intraclass correlation (ICC: twoway random effects model, single measure, absolute agreement) were $0.90,0.95$ and 0.92 for the for the ADL, performance and total TETRAS scores. Voller and colleagues found an test-retest ICC of 0.974 for the performance scale performed $15 \mathrm{~min}$ apart by the one rater ${ }^{10}$. These results need to be confirmed in a larger cohort of patients and with multiple examiners. Meanwhile, the data in Table 2 and these preliminary estimates of test-retest ICCs can be used

\begin{tabular}{|l|c|c|c|c|c|c|}
\hline & $\mathrm{N}$ & Mean & SD & Median & Minimum & Maximum \\
\hline ADL & 169 & 21.2 & 10.2 & 21.0 & 0.0 & 44.0 \\
\hline PERFORMANCE & 169 & 21.6 & 9.74 & 20.5 & 1.5 & 55.5 \\
\hline TOTAL SCORE & 169 & 43.0 & 18.8 & 42.0 & 3.0 & 99.5 \\
\hline AGE** & 169 & 62.9 & 15.0 & 64.0 & 15.0 & 99.0 \\
\hline \multicolumn{5}{|c|}{ TOTAL SCORE $<42 *$} \\
\hline ADL & 84 & 13.5 & 6.48 & 15.0 & 0.0 & 27.0 \\
\hline PERFORMANCE & 84 & 14.3 & 4.89 & 14.5 & 1.5 & 23.0 \\
\hline TOTAL SCORE & 84 & 27.7 & 9.51 & 29.0 & 3.0 & 41.5 \\
\hline AGE & 84 & 59.3 & 16.2 & 62.0 & 15.0 & 88.0 \\
\hline \multicolumn{7}{|c|}{ TOTAL SCORE $\geq 42 *$} \\
\hline ADL & 85 & 29.0 & 6.50 & 29.0 & 13.0 & 44.0 \\
\hline PERFORMANCE & 85 & 28.9 & 7.64 & 28.0 & 13.0 & 55.5 \\
\hline TOTAL SCORE & 85 & 57.9 & 12.6 & 56.5 & 42.0 & 99.5 \\
\hline AGE & 85 & 66.4 & 12.9 & 68.0 & 18.0 & 99.0 \\
\hline
\end{tabular}

*Statistics are given for the patients with total scores below the median (42) and greater than or equal to the median.

**Patient age in years

Table 2: TETRAS statistics for 169 patients with ET ( 85 men).

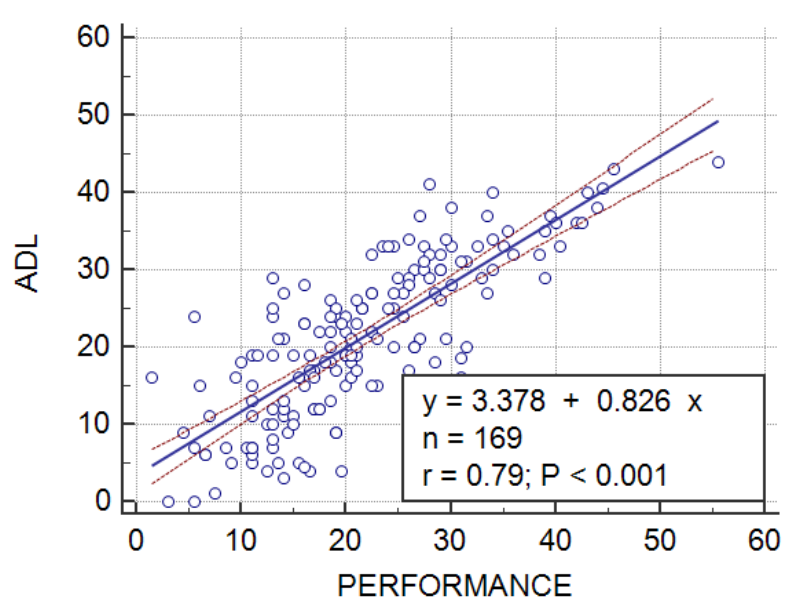

Figure 2: Linear regression and 95\% confidence limits for the ADL and performance TETRAS scores obtained from 169 patients with mild-severe ET ( 85 men).

in estimating sample sizes for parallel and crossover study designs. Intra-rater reliability for the TETRAS performance scale is greater than 0.9 , so most of the test-retest variability will be due to natural variability in the patients' tremor when multiple raters are involved, each patient being examined by a single rater. Test-retest reliability of the TETRAS ADL scale could be affected by many factors, including placebo effect and depression.

\section{Advantages and disadvantages of TETRAS vis-a-vis other scales}

TETRAS has been compared to other scales in a recent review $^{6}$. The FTM scale has been the scale most widely used in studies of ET, but TETRAS is gaining in popularity. TETRAS is better suited than FTM for patient populations with severe tremor because the tremor amplitude anchors for 0-4 ratings are much smaller in the FTM $(0$ : no tremor, 1: barely perceptible tremor, $2:<2 \mathrm{~cm}, 3: 2-4 \mathrm{~cm}$, and $4:>4$ $\mathrm{cm}$ ). Thus, grade 4 hand tremor in FTM part A might be as low as grade 2.5 in TETRAS (Table 1). This ceiling effect is a problem if the upper extremity ratings in FTM part A are used as a primary measure of tremor severity. Despite this ceiling effect, total FTM scores correlate strongly with total TETRAS scores (Figure 1).

TETRAS was designed for the rapid and valid assessment of ET severity. Its validity in other forms of tremor (dystonic tremor, Holmes tremor, Parkinson disease) has not been demonstrated. TETRAS is not well suited for focal and taskspecific tremors (writing tremor, orthostatic tremor).

TETRAS does not provide a comprehensive neurological characterization of patients with ET. Questionable signs of dystonia (e.g., mild head tilt), questionable bradykinesia, and impaired tandem walking are commonly seen in patients being evaluated for ET, and TETRAS will not capture these signs, which may be very important in differential diagnosis ${ }^{19}$. 


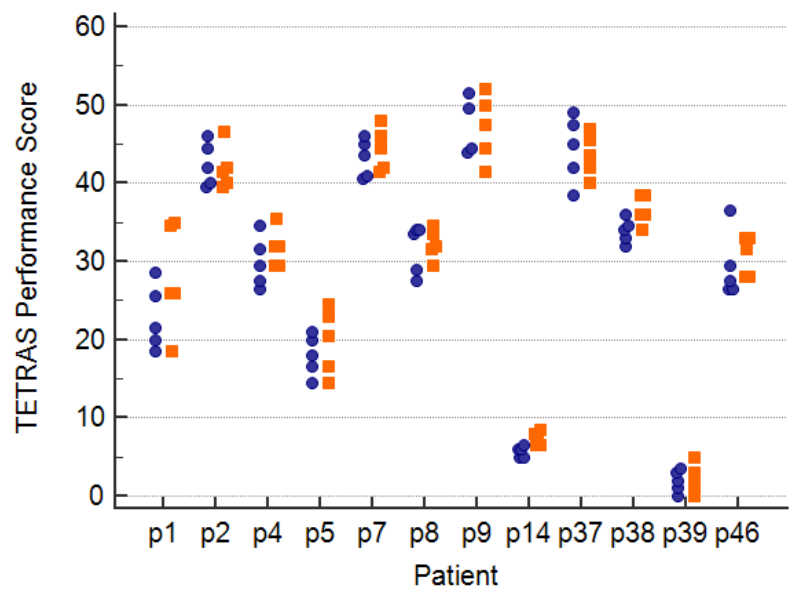

Figure 3: TETRAS performance scores for 10 ET patients and 2 normal controls, produced by 5 expert raters who developed TETRAS (blue circles) and by 5 neurology residents who had no training or experience in the use of TETRAS (orange squares).

A strength of TETRAS is that its anchors are very explicit, and many performance measures are based on estimates of peak-to-peak tremor amplitude, not on subjective anchors such as "normal, mild, moderate and severe", which are biased by the variable clinical experience of examiners. We compared the reliability of 5 neurology residents with 5 experienced movement disorder specialists in the completion of the TETRAS performance scale $^{20}$. The movement disorder specialists participated in the development of TETRAS, but the residents had no experience or training in TETRAS. The residents and experts rated 12 videos (10 ET patients and 2 normal people) twice, 1-2 months apart, to determine inter- and intra-rater reliability for the TETRAS performance section. The inter-rater intraclass correlations (two-way random effects model, single measure, absolute agreement) were 0.95 and 0.91 for the experts and residents, and the mean intra-rater reliability was 0.97 for the residents and experts. These data suggest that little or no training or experience is needed to achieve excellent reliability in TETRAS (Figure 3). By contrast, other popular tremor scales require training or reference manuals to achieve good reliability ${ }^{2,21,22}$.

\section{Individual and Commercial use of TETRAS}

TETRAS is copyrighted by TRG. Individual investigators and clinicians may use TETRAS free of charge. Commercial entities are charged a fee, paid to TRG. Questions regarding the commercial use of TETRAS should be directed to the president of TRG via e-mail (tremorresearchgroup@gmail. com).

\section{Acknowledgment}

This work was supported by the Spastic Paralysis Research Foundation of Kiwanis International, IllinoisEastern Iowa District.

\section{Conflicts of interest}

The author is a member of the Tremor Research Group, which holds the copyright on TETRAS.

\section{References}

1. Fahn S, Tolosa E, Marín C. Clinical rating scale for tremor. In: Jankovic J, Tolosa E, editors. Parkinson's Disease and Movement Disorders. 2nd ed. Baltimore: Williams \& Wilkins; 1993. p. 225-34.

2. Stacy MA, Elble RJ, Ondo WG, Wu SC, Hulihan J. Assessment of interrater and intrarater reliability of the Fahn-Tolosa-Marin Tremor Rating Scale in essential tremor. Mov Disord 2007 Apr 30;22(6):833-8.

3. Elble R, Comella C, Fahn S, Hallett M, Jankovic J, Juncos JL, et al Reliability of a new scale for essential tremor. Mov Disord 2012 Oct 2;27(12):1567-9. doi:10.1002/mds.25162.

4. Lorenz D, Poremba C, Papengut F, Schreiber S, Deuschl G. The psychosocial burden of essential tremor in an outpatient- and a community-based cohort. Eur J Neurol 2011 Jul;18(7):972-9. doi:10.1111/j.1468-1331.2010.03295.x.

5. Lorenz D, Schwieger D, Moises H, Deuschl G. Quality of life and personality in essential tremor patients. Mov Disord 2006 Aug;21(8):1114-8.

6. Elble R, Bain P, Forjaz MJ, Haubenberger D, Testa C, Goetz CG, et al. Task force report: scales for screening and evaluating tremor: critique and recommendations. Mov Disord 2013 Nov;28(13):1793-800. doi:10.1002/mds.25648.

7. Papengut F, Raethjen J, Binder A, Deuschl G. Rest tremor suppression may separate essential from parkinsonian rest tremor. Parkinsonism Relat Disord 2013 Jul;19(7):693-7. doi:10.1016/j. parkreldis.2013.03.013.

8. Uchida K, Hirayama M, Yamashita F, Hori N, Nakamura T, Sobue G. Tremor is attenuated during walking in essential tremor with resting tremor but not parkinsonian tremor. J Clin Neurosci 2011 Sep;18(9):1224-8. doi:S0967-5868(11)00169-X [pii] 10.1016/j. jocn.2010.12.053.

9. Mostile G, Giuffrida JP, Adam OR, Davidson A, Jankovic J. Correlation between Kinesia system assessments and clinical tremor scores in patients with essential tremor. Mov Disord 2010 Jul 8;25(12):1938-43.

10.Voller B, Lines E, McCrossin G, Artiles A, Tinaz S, Lungu C, et al. Alcohol challenge and sensitivity to change of the essential tremor rating assessment scale. Mov Disord 2014 Apr;29(4):555-8. doi:10.1002/ mds. 25667.

11. Elble R, Hellriegel H, Raethjen J, Deuschl G. Assessment of head tremor with accelerometers versus gyroscopic transducers. Movement Disorders Clinical Practice 2016;(in press). doi:10.1002/mdc3.12379.

12. Mostile G, Fekete R, Giuffrida JP, Yaltho T, Davidson A, Nicoletti A, et al. Amplitude fluctuations in essential tremor. Parkinsonism Relat Disord 2012 May 14;18(7):859-63. doi:10.1016/j.parkreldis.2012.04.019.

13. Akano E, Zesiewicz T, Elble R. Fahn-Tolosa-Marin scale, digitizing tablet and accelerometry have comparable minimum detectable change. Mov Disord 2015;30(Suppl 1):S556.

14.Elble R. Digitizing tablet and Fahn-Tolosa-Marín tremor ratings have comparable minimum detectable change. Mov Disord 2016;31.

15.Elble R, Zesiewicz T. Fahn-Tolosa-Marin tremor scale and digitizing tablet have comparable minimum detectable change. Mov Disord 2015;30(Suppl 1):S558.

16. Elble RJ, McNames J. Using Portable Transducers to Measure Tremor Severity. Tremor Other Hyperkinet Mov (N Y) 2016;6:375. doi:10.7916/D8DR2VCC.

17.Chang WS, Chung JC, Kim JP, Chang JW. Simultaneous Thalamic and 
Posterior Subthalamic Electrode Insertion With Single Deep Brain Stimulation Electrode for Essential Tremor. Neuromodulation 2012 Sep 17. doi:10.1111/j.1525-1403.2012.00503.x.

18. Deuschl G, Bain P, Brin M. Consensus statement of the Movement Disorder Society on Tremor. Ad Hoc Scientific Committee. Mov Disord 1998;13(Suppl 3):2-23.

19. Elble RJ. The essential tremor syndromes. Curr Opin Neurol 2016 Jun 1. doi:10.1097/WCO.0000000000000347.
20.Elble R, Bhargava P, Dhakal L, Jalan P, Kaul S, Pandav V, et al. Reliability of the Essential Tremor Rating Assessment Scale in untrained neurology residents Neurology 2013;80(7 Supplement):S53.006.

21.Bain PG, Findley LJ. Assessing Tremor Severity: A Clinical Handbook. London: Smith-Gordon; 1993.

22.Louis ED, Barnes L, Wendt KJ, Ford B, Sangiorgio M, Tabbal S, et al. A teaching videotape for the assessment of essential tremor. Mov Disord 2001;16(1):89-93. 